International Journal of Engineering \&Technology, $7(3.12)(2018) 392-402$
SPC
International Journal of Engineering \& Technology
Website: www.sciencepubco.com/index.php/IJET
Research paper

\title{
Selection of Industrial Robots for Automation Applications in Multiple Attribute Decision Making Environment using the Analytical Network Process
}

\author{
N.Rishi Kanth ${ }^{1}$, A.Srinath ${ }^{2}$, J. Suresh Kumar ${ }^{3}$ \\ ${ }^{1}$ Associate Professor, Sri Chundi Ranganayakulu Engineering College. \\ ${ }^{2}$ Professor \& Head, Mechanical Engineering KL University, Vijayawada \\ ${ }^{3}$ Professor \& Head, Mechanical Engineering, JNTUH College of Engineering, Hyderabad (Autonomous) \\ *Corresponding Author Email: ${ }^{1}$ Nuthi.rishi@gmail.com
}

\begin{abstract}
Analytical Network process (ANP), is applied here as a decision making technique for the selection of appropriate robots for industrial and automation applications. The core motivation of applying, in particular, the ANP technique is that robot selection is dependent upon a number of attributes and criteria which have strong influences/interdependencies upon each other. The ANP, as a multiple attribute decision making (MADM) technique for robot selection, captures the effects of these cross hierarchical dependencies, and appropriately maps the influences within the clusters and between the various alternatives. Simultaneously, the technique does not include the assumption of independence of higher-level elements from lower level elements and about the independence of the elements within a level. First, a set of attributes, which influence the selection of the robots, are identified. Next, using the various steps of ANP, viz., pair wise comparisons matrices and priority vectors determination and the development of the super-matrix the global weights of the attributes with respect to other attributes are determined. The final alternatives are then rated as per the graduated weights of the respective attributes. Thus, a comprehensive solution towards selection of robots enabling the decision-makers to suitably understand the complex relationships of the relevant qualitative and quantitative attributes in the decision-making is obtained. The technique is also illustrated using detailed analysis for a specific case of decision making between three robot suppliers and selection of appropriate robot from alternatives. In order to get more insight into relationships among various attributes and their effect on decision makers, the sensitivity analysis of the results with respect to determinant level attributes is carried out.
\end{abstract}

Keywords: Analytical Network process (ANP), AHP, Robot selection, priority vector, super matrix, sensitivity analysis.

\section{Introduction}

Industries across the world, in recent years, are witnessing a momentous shift from the conventional manufacturing processes towards the automation and flexible mechanization systems. Indeed, in order to increase the productivity, accuracy and precision in operations, the automation systems are rapidly replacing the manual systems and correspondingly reducing the overall human intervention. Most often, these automation solutions include robots and integrated robotic applications. Robotic systems, along with the aforesaid advantages, also lead to reduction in monotony and risk factors. However, the overal gains achieved using robots, in terms of increase in productivity of operations, is largely dependent on the appropriate selection of the particular robot for the application concern. Consequently, the selection of appropriate robot, from the vast spectrum of the various robots and their models from various manufacturers available today, for the particular application in hand becomes one of the most crucial decision making exercise for the modern system designers.

\subsection{Analytical Network Process}

ANP is particularly useful in decision making problems which include cross hierarchical dependencies, influences within the clusters and between the alternatives and does not necessarily include the assumption of independence of higher-level elements from lower level elements and about the independence of the elements within a level. Figure 1 shows a schematic representation of a network in 1(a) which involves feedbacks, interdependencies and cross hierarchical influences as compared to a hierarchical structure, in 1(b), used in AHP.

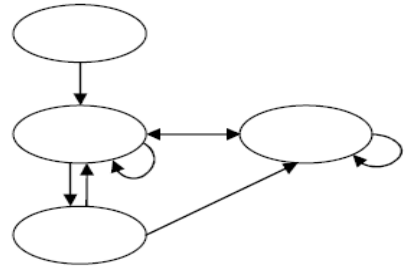

(a) a Network

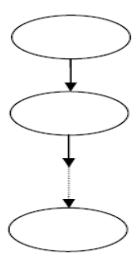

(b) a Hieraricy
Fig. 1: Structural Difference between a Network and Hierarchy

It can be seen that the AHP is a special case of ANP, methodology. Note that, the nodes represent the levels in hierarchy and components in the network.

In the present work, the methodology of ANP is used for decision making problem of selection of robots for industrial and automation systems. The ANP is essentially the best suitable 
decision making tool in robot selection because, as enumerated in literature, various subjective and objective criteria and parameters are involved in robot selection and most of them have complicated cross dependencies across the hierarchies. Besides, there exist a loose/conditional correlation also between some elements and criteria in the robot selection. Since, both these things, viz., intradependencies and conditional correlation of criteria, can be modelled appropriately in ANP only, ANP is presented here as an efficient technique for decision making and selection of robots.

\section{Robot Selection Using ANP Technique}

In this section, the application of ANP methodology for selection of robot for industrial applications in a multi-criteria decisionmaking environment is presented.

\subsection{Identification of Attributes/Parameters which Influence Robot Selection}

First a set of criteria which influence the robot selection are identified. A strategic classification of the various attribute and their clusters influencing the decision making in robot selection is carried out. The criteria which play a crucial role in the decisionmaking and, at the same time, are dependent on the remaining criteria, have been named as determinants. These are listed in Section 3.1. The second tier of the classification encompasses the clusters supporting the realization of the upper-level decisive factors. The second tier criteria are named variously as clusters, norms, dimensions etc. In addition to this, the clusters/norms are separately dependent on some other sub-criteria, which have been named as facilitators. The third tier comparisons of these facilitators/enablers.

\subsubsection{Attributes Identification-Determinant Level}

Various determinant level parameters and attributes which influence the selection of robot, are identified as:

(i) Scope available for customization: Robot investments often have to make a trade off between conflicting requirements. In such a scenario the robot which is finally selected/installed as a part of automation solution must meet the conflicting requirements in terms of process specifications, electrical requirements etc. Hence scope of customization available to the end-user is one of the most important selection criteria.

(ii) Worth: The value and worth of the robot has to be seen in more comprehensive terms here wherein in addition to the cost of the robot it involves service support, delivery schedules, availability of spare parts, network support etc. All these criteria are definitely of high importance in robot selection process. (iii) Quality: This criteria envelopes the considerations involving resolution, accuracy, precision, mean time between failure (MTBF), mean time to repair (MTTR), etc.

(iv) Performance: The overall working parameters of the robot including its speed, maximum overload capacity, precision, manipulability, dextricity etc. and form high criteria for considerations for robotic selection.

(v) Compatibility of robot with the existing set up at installation site: It may be important to assess the role of compatibility criteria which includes the influence of existing set-up at the customer end on his decision choices. Thus, eg., if a particular manufacturing company has a set of robots of company A (say), and it is planning for expansion then there are high chances of manufacturing company selecting the robots of company A as it provides leverage in compatibility with existing set up of power supply, electrical controls, spares maintenance etc. Thus this criteria is also of high importance.

The above five criteria will be taken up as five determinants for estimating the overall weighted index (OWI) for the various providers of robots.

\subsubsection{Attributes Identification-Cluster/Norm Level}

The five determinant level criteria in-turn are influenced by the various clusters of attributes. These sub-criteria which support and influence the determinant level criteria, as identified by Aggarwal et al. [13], are as follows:

i. Configuration of robot;

ii. Electrical controls;

iii. Geometric constraints and limitations;

iv. Repeatability;

The above parameters are however based on technical specifications of the robotic systems. Hence, to take into account the commercial and installation aspects of the robotic systems, the following attributes are added to the list:

v. Reliability;

vi. Economic viability.

\subsubsection{Sub-Cluster-Norm Level Criteria-Facilitators/ Enablers}

The criteria and attribute clusters are broken down into their subelements. This is essential because in addition to the inter-cluster influences there exists intra-attributes influences. The overall influence on the decision is dependent on the sum total of these effects. Various sub-elements of the clusters and their relevance in robot selection along with appropriate references are presented in Table 1.

Table 1: Various enabler/facilitator level criteria and their relevance in robot selection

\begin{tabular}{|c|c|c|}
\hline S. No. & Criteria & Relevance \\
\hline 1 & Payload capacity (PC) & $\begin{array}{l}\text { It refers to the specified ability of the robot to lift maximum payload at its extreme horizontal/vertical reaches. It } \\
\text { is important that the selected robot can handle the payload applied during working cycle in the given range. }\end{array}$ \\
\hline 2 & Workspace (WS) & $\begin{array}{l}\text { It is the maximum horizontal and vertical reach of the robot to constitute its envelope. It is essential to ensure } \\
\text { that desired area of operations and movements of tool tip attached at robot is within the robot envelop. }\end{array}$ \\
\hline 3 & Overload capacity (OC) & $\begin{array}{l}\text { The robot must be able to handle minor cyclic fluctuations in the end payload. Thus the overload capacity of } \\
\text { robot is an essential consideration for its selection for a job at hand. }\end{array}$ \\
\hline 4 & Accuracy (AC) & $\begin{array}{l}\text { It specifies the minimum error with which a robot can reach a commanded position. It may vary with speed and } \\
\text { position within the working envelope and with payload and hence must be considered as selection criteria. }\end{array}$ \\
\hline 5 & Resolution (RS) & $\begin{array}{l}\text { The lower and above least counts of robot working parameters must be checked to ensure their applicability in } \\
\text { the robot working range. }\end{array}$ \\
\hline 6 & $\begin{array}{l}\text { Computational efficiency } \\
\text { (CE) }\end{array}$ & $\begin{array}{l}\text { It determines the speed of processing and calculations to make the operations and controlling of robot real time. } \\
\text { However, there is a trade off between the computational efficiency, processing speed and real time control and } \\
\text { the cost of the algorithm and the controller. }\end{array}$ \\
\hline 7 & Manipulability (MA) & $\begin{array}{l}\text { The skillful and possible movements and orientations of the links/joints of robot gives it versatility and increases } \\
\text { its working utility. Manipulability thus becomes important consideration for robot selection. }\end{array}$ \\
\hline 8 & Joint type (JT) & $\begin{array}{l}\text { The kinematics details including the link and joint types influences many criteria including payload, workspace, } \\
\text { manipulability, etc. }\end{array}$ \\
\hline 9 & Power source req. (PR) & $\begin{array}{l}\text { The power specifications required by robot, viz. separate earthing for welding robots, uniform voltage for } \\
\text { painting robots must be available at the installation site of the robot. Else the robot may not work optimally. }\end{array}$ \\
\hline
\end{tabular}




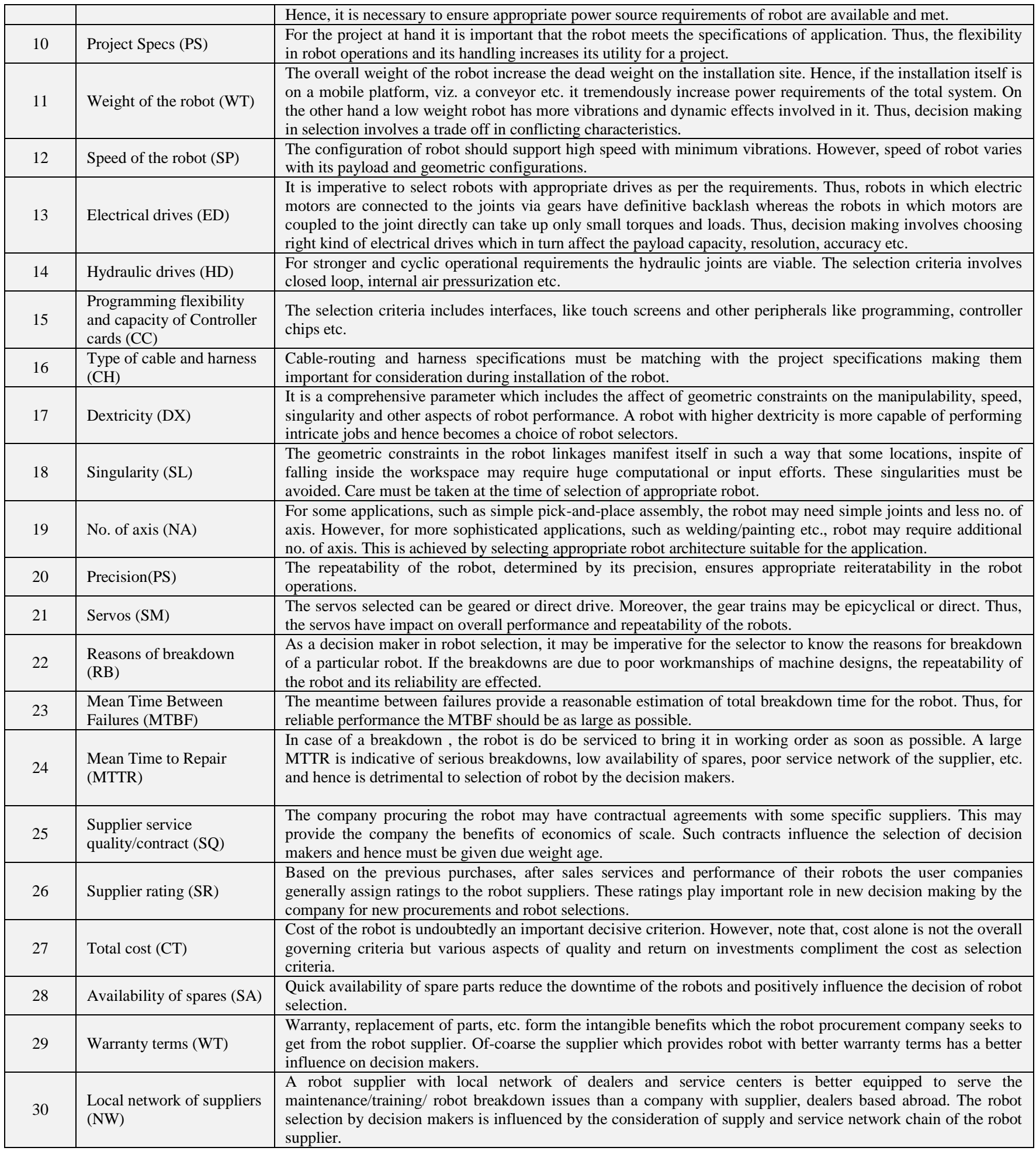

\subsection{ANP Model Formulation}

ANP based model formulation for robot selection is done using the three tier classification presented above. Thus, a network is established wherein the robot selection is based upon the overall weighted index (OWI) of the various suppliers. The OWI is scored based upon the comparative influence of the determinants. The determinant attributes are influenced by the clusters which are in turn influenced by the facilitators. Moreover, note that, the facilitators not only influence the respective clusters but also have strong interdependencies and support among.

themselves. Mapping of the influence of each of the attributes upon the other parameters (higher in hierarchy or at the same level in the network) is done in ANP model and a looped arc is used to show such interdependencies within the same level of analysis. Figure 3 shows the ANP based model for robot selection in multiple attribute based decision making environment. Note that, the model is prepared with respect to three suppliers of robots, namely, A, B, and C. While in actual analysis the names corresponded to the actual companies whose products (Robots) were compared with each other and using whom the pair-wise comparisons were done, the actual names of the company are not disclosed here. Moreover, for the purpose of brevity, the results corresponding only to the scope of customization determinant are presented in detail. However, the results corresponding to all the five determinants would be used in the calculation of overall weighted index (OWI), which indicates the score of a robot supplier after considering all the criteria as included in the ANP model. 


\subsection{Pair-wise Comparisons of the Determinants}

In this step the attributes and decision elements at determinant level are compared pair-wise with respect to their importance towards the attainment of objective (robot selection). Each determinant level criterion is pair-wise compared with other determinant level criteria with respect to the contribution to the goal.

It will suffice here to outline the technique adopted, in this paper, to obtain the pairwise comparative rating between two criteria, at any level of classification, namely determinant, norm and facilitators. In order to get the genuine ratings of each parameter viz-a-viz other parameters, a three pronged strategy has been adopted. First a set of request proposals have been developed wherein a brief description of various attributes (at various levels), as presented in Section 3.1, are put in a comparative matrix format The questions are then given to a panel of experts on the subject who are requested to rate them pairwise. The rating/relative importance values are determined with Saaty's 1-9 scale, where a score of 1 represents equal importance between the two elements and a score of 9 indicates the extreme importance of one element (row component in the matrix) compared to the other one (column component in the matrix). A reciprocal value is assigned to the inverse comparison; that is, aij=1/aji, where aij (aji) denotes the importance of the ith (jth) element.

As the third part of the strategy for determination of pair-wise ratings, the geometric mean of the various ratings, between two given criteria, given by different experts is taken. The geometric mean, then, represents the pair-wise comparative value to be taken for the determination of priority vectors between two elements.

Since the pairwise comparison matrices are formed using the group decision making followed by geometric averaging of individual judgments, the effect of the individual biases have been eliminated from the analysis. (i) consensus, voting in groups, and (iii) geometric mean of the individual's judgments as methods to include objective opinion rankings and eliminate individual biases from the analysis.

Note that, similar procedure is adopted to get the weighted rankings/values of pairwise comparisons between attributes at cluster-norm and facilitator level.

The local priority vectors are determined by solving the equation

$\mathrm{A} \times \mathrm{w}=\lambda \max \times \mathrm{w}$

where $\mathrm{A}$ is the matrix of pairwise comparison, $\mathrm{w}$ is the eigenvector, and $\lambda \max$ is the largest eigenvalue of $A$. In this paper, the following three-step procedure is used to determine the priority vectors

1. Sum the values in each column of the pairwise comparison matrix;

2. Divide each element in a column by the sum of its respective column. The resultant matrix s referred to as the normalized pairwise comparison matrix;

3. Sum the elements in each row of the normalized pairwise comparison matrix, and divide the sum by the $\mathrm{n}$ elements in the row.

Table 2: Pairwise comparisons of determinants $(\mathrm{CR}=8.28)$

\begin{tabular}{|c|c|c|c|c|c|c|}
\hline & SC & WTH & QLT & PER & COM & Priority vector \\
\hline Scope of Customization (SC) & 1 & 3 & 2 & $1 / 2$ & $1 / 2$ & 0.1946 \\
\hline Worth (WTH) & $1 / 3$ & 1 & 4 & $1 / 2$ & $1 / 2$ & 0.1541 \\
\hline Quality (QLT) & $1 / 2$ & $1 / 4$ & 1 & $1 / 2$ & $1 / 4$ & 0.0761 \\
\hline Compatibility (COM) & 2 & 2 & 4 & 3 & 1 & 0.3651 \\
\hline
\end{tabular}

These final numbers provide an estimate of the relative priorities for the elements being compared with respect to its upper level criterion. The resultant set is priority vector associated with the pairwise comparison matrix. Note that, priority vectors must be derived for all comparison matrices. The priority vectors from pairwise comparisons of determinants, shown in Table-2, will be used for the calculation of overall weighted index (OWI).

towards the attainment of objective (robot selection). The methodology of obtaining the relative pair-wise comparison values and the evaluation of priority vectors is similar to the one outlined in Section 3.3. Since there are six cluster level norms and five determinants, there will be five 6x6 dimanetional matrices, one for the each determinant. The matrix for the scope of customization (SC) determinant is shown in Table 3. The results of this comparison (priority vectors) are carried as Pja in Table 9.

\subsection{Pair-wise Comparisons of the Cluster/Norm Level Attributes}

In this step the attributes and decision elements at the cluster norm level are compared pair-wise with respect to their importance

Table 3: Pairwise comparisons of cluster level norm attributes $(\mathrm{CR}=5.94)$

\begin{tabular}{|c|c|c|c|c|c|c|c|}
\hline & CR & EC & GC & $\mathbf{R P}$ & $\mathbf{R L}$ & EV & Priority vector \\
\hline Configuration of robot $(\mathrm{CR})$ & 1 & 3 & 2 & $1 / 2$ & 2 & 2 & 0.2123 \\
\hline Electrical and Controls (EC) & $1 / 3$ & 1 & $1 / 2$ & $1 / 3$ & $1 / 4$ & $1 / 3$ & 0.0594 \\
\hline Geometric constraints (GC) & $1 / 2$ & 2 & 1 & $1 / 2$ & $1 / 3$ & $1 / 4$ & 0.0917 \\
\hline Reliability (RL) & $1 / 2$ & 4 & 3 & $1 / 2$ & 1 & 2 & 0.1914 \\
\hline Economic Viability (EV) & $1 / 2$ & 3 & 4 & $1 / 3$ & $1 / 2$ & 1 & 0.1524 \\
\hline
\end{tabular}

In this step, the pairwise comparison of attributes and parameters at the facilitator level is conducted with respect to their relative influences towards their controlling criterion. Thus, pairwise comparison for a determinant is done among the applicable enablers within a given dimension cluster. The number of such pairwise comparison matrices depends on the number of determinants and dimensions in the ANP model. In the present case, there are five parameters at determinant level and six dimensions at cluster norms level. Hence, in total there will be 30 (=6x5) number of matrices. One such pairwise comparison matrix for Configuration of robot (CR) dimension under Scope of customization (SC) determinant is shown in Table 4. For the pairwise comparison, the question asked to the decision-maker is: "what is the relative impact on the configuration of robot by enabler a (say payload) when compared to enabler b (say workspace) in improving scope of customization available to the robot user and the supplier?"

It is observed from Table 4 that the relative importance of PC when compared to WS with respect to Configuration of robot in achieving increment in scope of customization of robotic solution is 2.5 
Table 4: Pairwise comparisons of facilitator level attributes mapping their combined effect of configuration of root under scope of customization determinant $(\mathrm{CR}=9.11)$

\begin{tabular}{|l|c|c|c|c|c|c|c|c|c|c|c|c|c|}
\hline & PC & WS & OC & AC & RS & CE & MA & JT & PR & PS & WR & SP & PV \\
\hline PC & 1 & 2 & $1 / 2$ & 3 & 2 & 4 & $1 / 3$ & $1 / 3$ & 2 & $1 / 4$ & $1 / 2$ & $1 / 2$ & $\mathbf{0 . 0 6 5 6}$ \\
\hline WS & $1 / 2$ & 1 & $1 / 2$ & 2 & $1 / 2$ & 3 & $1 / 4$ & $1 / 2$ & 3 & $1 / 3$ & $1 / 3$ & $1 / 4$ & $\mathbf{0 . 0 5 1 5}$ \\
\hline OC & 2 & 2 & 1 & 2 & 2 & 4 & 1 & 3 & 4 & $1 / 2$ & 3 \\
\hline AC & $1 / 3$ & $1 / 2$ & $1 / 2$ & 1 & 2 & 4 & $1 / 4$ & $1 / 2$ & 2 & $1 / 4$ & 3 & $1 / 3$ & $\mathbf{0 . 1 3 1 7}$ \\
\hline RS & $1 / 2$ & 2 & $1 / 2$ & $1 / 2$ & 1 & 4 & $1 / 3$ & $1 / 3$ & 2 & $1 / 4$ & 2 & $1 / 3$ & $\mathbf{0 . 0 5 4 7}$ \\
\hline CE & $1 / 4$ & $1 / 3$ & $1 / 4$ & $1 / 4$ & $1 / 4$ & 1 & $1 / 4$ & $1 / 3$ & $1 / 4$ & $1 / 4$ & $1 / 4$ & $1 / 4$ & $\mathbf{0 . 0 2 1 2}$ \\
\hline MA & 3 & 4 & 1 & 4 & 3 & 4 & 1 & 2 & 2 & 2 & 4 & 3 & $\mathbf{0 . 1 6 4 1}$ \\
\hline JT & 3 & 2 & $1 / 3$ & 2 & 3 & 3 & $1 / 2$ & 1 & 1 & $1 / 3$ & 2 & 2 & $\mathbf{0 . 0 8 7 4}$ \\
\hline PR & $1 / 2$ & $1 / 3$ & $1 / 4$ & $1 / 2$ & $1 / 2$ & 4 & $1 / 2$ & 1 & 1 & $1 / 4$ & 2 & 3 & $\mathbf{0 . 0 6 1 1}$ \\
\hline PS & 4 & 3 & 2 & 4 & 4 & 4 & $1 / 2$ & 3 & 4 & 1 & 4 & 2 & $\mathbf{0 . 1 6 7 5}$ \\
\hline WR & 2 & 3 & $1 / 3$ & $1 / 3$ & $1 / 2$ & 4 & $1 / 4$ & $1 / 2$ & $1 / 2$ & $1 / 4$ & 1 & $1 / 2$ & $\mathbf{0 . 0 5 1 9}$ \\
\hline SP & 2 & 4 & $1 / 3$ & 3 & 3 & 4 & $1 / 3$ & $1 / 2$ & $1 / 3$ & $1 / 2$ & 2 & 1 & $\mathbf{0 . 0 8 4 8}$ \\
\hline
\end{tabular}

It is also observed from Table 4 that enabler PS, MA and OC of robots have higher influence $(0.1675,0.1641$ and 0.1317 respectively) on configuration of robot in improving Scope of customization between the robot user and the supplier. Similarly, computational efficiency (CE) has the least influence (0.0212) on the robot configuration in improving the scope of customization. The priority-vectors obtained from these matrices will be used in Super matrix determination.

\subsection{Pair-wise Comparisons for Interdependencies among the Facilitators/Enabler Attributes}

In this step, the interdependencies of the attributes at facilitator level are mapped by doing the pairwise comparisons among themselves. Thus, the pairwise comparison of attributes and parameters at the facilitator level is conducted to capture the effect of one controlling facilitator/enabler level attribute on the other enabler attributes in a particular cluster and determinant group The number of such pairwise comparison matrices depends on the number of determinants and dimensions in the ANP model. One such pairwise comparison matrix for mapping the effects of configuration of robot (CR) dimension under Scope of customization (SC) determinant with payload capacity (PC) as the controlling criteria on the other attributes at facilitator level in the same cluster is shown in Table 5. For the pairwise comparison, the question asked to the decision-maker is: "when considering payload capacity with regard to increasing scope of customization, what is the relative impact on the enabler a (say workspace) when compared to enabler b (say overload capacity) in the given configuration of robot cluster?" The method of rating the attributes is similar to that used in Section In accordance with the network.

It is observed that workspace (WS) has the maximum impact (0.1826) on the Scope of customization and Robot configuration cluster with payload capacity (PC) as the control enabler attribute. Note that in total there will be 150 such matrices for the given model. The priority vectors from these matrices are used in the formation of a super-matrix.

Table 5: Pairwise comparison matrix for facilitator level attributes under scope of customization, configuration of robot and payload capacity (CR=8.9)

\begin{tabular}{|l|c|c|c|c|c|c|c|c|c|c|c|c|}
\hline & WS & OC & AC & RS & CE & MA & JT & PR & PS & WR & SP & Priority vector \\
\hline WS & 1 & 2 & 4 & 5 & 2 & 2 & 3 & 2 & 4 & 2 & 3 & $\mathbf{0 . 1 8 2 6 4 5}$ \\
\hline OC & $1 / 2$ & 1 & 3 & 2 & 2 & 5 & 3 & 2 & 3 & 2 & 3 & $\mathbf{0 . 1 6 0 1 6 2}$ \\
\hline AC & $1 / 4$ & $1 / 3$ & 1 & 2 & $1 / 2$ & $1 / 2$ & $1 / 3$ & 0.2 & $1 / 2$ & $1 / 2$ & $1 / 4$ & $\mathbf{0 . 0 3 5 4 9 5}$ \\
\hline RS & 0.2 & $1 / 2$ & $1 / 2$ & 1 & $1 / 2$ & $1 / 3$ & $1 / 2$ & $1 / 3$ & $1 / 2$ & $1 / 2$ & $1 / 2$ & $\mathbf{0 . 0 3 4 5 5 6}$ \\
\hline CE & $1 / 2$ & $1 / 2$ & 2 & 2 & 1 & 2 & 4 & 2 & 2 & 4 & $1 / 4$ & $\mathbf{0 . 1 0 7 2 0 4}$ \\
\hline MA & $1 / 2$ & 0.2 & 2 & 3 & $1 / 2$ & 1 & 2 & 3 & 2 & 4 & $1 / 2$ & $\mathbf{0 . 0 9 3 5 0 2}$ \\
\hline JT & $1 / 3$ & $1 / 3$ & 3 & 2 & $1 / 4$ & $1 / 2$ & 1 & 4 & 2 & 4 & $1 / 3$ & $\mathbf{0 . 0 8 5 2 7 3}$ \\
\hline PR & $1 / 2$ & $1 / 2$ & 5 & 3 & $1 / 2$ & $1 / 3$ & $1 / 4$ & 1 & 2 & 2 & $1 / 2$ & $\mathbf{0 . 0 7 6 0 3}$ \\
\hline PS & $1 / 4$ & $1 / 3$ & 2 & 2 & $1 / 2$ & $1 / 2$ & $1 / 4$ & $1 / 2$ & 1 & 2 & $1 / 3$ & $\mathbf{0 . 0 4 8 4 4 1}$ \\
\hline WR & $1 / 2$ & $1 / 2$ & 2 & 2 & $1 / 4$ & $1 / 4$ & $1 / 4$ & $1 / 2$ & $1 / 2$ & 1 & $1 / 2$ & $\mathbf{0 . 0 4 7 5 3 3}$ \\
\hline SP & $1 / 3$ & $1 / 3$ & 4 & 2 & 4 & 2 & 3 & 2 & 3 & 2 & 1 & $\mathbf{0 . 1 2 9 1 5 9}$ \\
\hline
\end{tabular}

\subsection{Evaluation of Providers}

The last pairwise comparison matrix is generated by comparative evaluation of the alternative suppliers of the robot. In the present analysis three different robotic suppliers were shortlisted. The pairwise comparisons of these alternative suppliers (namely, A, B and $\mathrm{C}$ ) were done to map their relative impact on each of the facilitator level attribute. The number of such pairwise comparison matrices depend upon the number of determinant level attributes and the number of attributes in the model at the facilitator level in the model. Thus, since in the present model, there are five determinant level criteria and in total thirty enabler level attributes, total number of matrices formed are $150(=30 \times 5)$. For brevity, the pairwise comparison matrix between $\mathrm{A}, \mathrm{B}$ and $\mathrm{C}$ with respect to the payload capacity of their robots and the relative impact with respect to scope of customization in robot is shown in Table 6 The question asked to the decision makers is, "what is the impact of the three alternatives, A, B and C on the enabler payload in influencing the determinant Scope of customization. The priority vector from this matrix is used in the compatibility desirability matrix formation.

Table 6: Matrix for alternatives' impact on enabler Payload capacity (PC) in influencing the Scope of customization determinant (CR=7.6)

\begin{tabular}{|c|c|c|c|c|}
\hline & A & B & C & Priority vector \\
\hline A & 1 & $1 / 2$ & 3 & $\mathbf{0 . 3 5 0 0}$ \\
\hline B & 2 & 1 & 2 & $\mathbf{0 . 4 7 7 8}$ \\
\hline C & $1 / 3$ & $1 / 2$ & 1 & $\mathbf{0 . 1 7 2 2}$ \\
\hline
\end{tabular}

\subsection{Super Matrix Formation}

In this step, a super-matrix, which synthesizes the priority vectors from various pair-wise comparison matrices is formed for overall criteria prioritization and alternative rankings. A super-matrix is a partitioned matrix where each sub-matrix is composed of a set of relationships between and within the levels as represented by the decision-maker's model. Thus the priority vectors from the pairwise comparison matrices are used to determine the overall relative importance of the enablers for each of the determinants. 


\subsection{Assortment of Data and the Desirability Indices Matrices}

Using the information inform of pairwise comparative scores among priority vectors, and comprehensive comparative scores from the super-matrix and limit super-matrix, the selection of best alternative is done. First, the selection of best alternative corresponding to each determinant is done,. For this the desirability indices, indicating the relative importance of the alternatives in supporting a particular determinant, are obtained corresponding to each determinant. The desirability index, $D_{i a}$ for alternative $i(=\mathrm{A}, \mathrm{B}$ or $\mathrm{C}$ in the present model) and the determinant $a$, is defined as [43]:

$$
D_{i a}=\sum_{j=i}^{J} \sum_{k=1}^{K_{j a}} P_{j a} A_{k j a}^{D} A_{k j a}^{I} S_{i k j a}
$$

where $P_{j a}$ is the relative importance weight of dimension $j$ in influencing the determinant $a$ which is obtained from the priority vector of the pairwise comparison matrices of cluster level norm attributes; $A_{k j a}^{D}$ is the relative importance weight for attribute at facilitator/enabler level and is obtained as the priority vector from pairwise comparison matrices of facilitator level attributes mapping their combined effect of various dimensions under scope of various determinants; ' $k$ ' in influencing the determinant a through cluster dimension $\mathrm{j}$ for dependency (D) relationships; $A_{k j a}^{I}$ is the stabilized relative importance weight for attribute enabler $k$ in the dimension $j$ and the determinant $a$ cluster for interdependency (I) relationships. These values are taken from converged super-matrix. Moreover, $S_{i k j a}$ is the relative impact of alternative $i$ on enabler $k$ of dimension $j$ for determinant $a$; $K_{j a}$ is the index set of attribute enablers for dimension $j$ of determinant $a$, $J$ is the index set of the cluster $j$.

Since in the ANP model, Figure 1, taken in this paper, five determinants are considered, the number of such desirability indices set $\boldsymbol{D}_{\boldsymbol{i}}$ ( $i=\mathrm{A}, \mathrm{B}$ and C) will be five. Table 9 shows the desirability indices for one determinant, the scope of customization, $D_{i(S C)}$. The desirability indices $D_{i(S C)}$ and the normalized desirability indices, $D_{i(S C)}$, for the three alternatives (A, $\mathrm{B}$ and $\mathrm{C}$ ) with respect to the scope of customization (SC) available in their robots are obtained using the eq. 1, as shown in Table 7.

Table 7: Desirability matrix for scope of customization

\begin{tabular}{|c|c|c|c|c|c|c|c|c|c|c|}
\hline \multirow{2}{*}{ Dimension } & & \multirow{2}{*}{$\boldsymbol{P}_{j(S C)}$} & \multirow{2}{*}{$A_{k j(S C)}^{D}$} & \multirow{2}{*}{$A_{k j(S C)}^{I}$} & \multicolumn{3}{|c|}{$S_{i k j(S C)}$} & \multicolumn{3}{|c|}{ Desirability index $D_{i(S C)}$. } \\
\hline & & & & & $\mathbf{A}$ & B & $\mathbf{C}$ & $i=\mathrm{A}$ & $I=B$ & $i=\mathrm{C}$ \\
\hline \multirow{12}{*}{$\begin{array}{l}\text { Configuration } \\
\text { of robot }\end{array}$} & PC & 0.212 & 0.066 & 0.093 & 0.35 & 0.478 & 0.172 & 0.000455 & 0.000622 & 0.000224 \\
\hline & WS & 0.212 & 0.052 & 0.091 & 0.557 & 0.32 & 0.123 & 0.000553 & 0.000318 & 0.000122 \\
\hline & OC & 0.212 & 0.132 & 0.077 & 0.517 & 0.359 & 0.124 & 0.001114 & 0.000773 & 0.000268 \\
\hline & $\mathbf{A C}$ & 0.212 & 0.058 & 0.065 & 0.35 & 0.478 & 0.172 & 0.00028 & 0.000382 & 0.000138 \\
\hline & $\mathbf{R S}$ & 0.212 & 0.055 & 0.076 & 0.49 & 0.312 & 0.198 & 0.000436 & 0.000277 & 0.000176 \\
\hline & $\mathbf{C E}$ & 0.212 & 0.021 & 0.075 & 0.531 & 0.322 & 0.147 & 0.000179 & 0.000109 & $4.96 \mathrm{E}-05$ \\
\hline & MA & 0.212 & 0.164 & 0.081 & 0.277 & 0.552 & 0.172 & 0.00078 & 0.001555 & 0.000484 \\
\hline & JT & 0.212 & 0.087 & 0.087 & 0.297 & 0.539 & 0.164 & 0.000482 & 0.000875 & 0.000266 \\
\hline & PR & 0.212 & 0.061 & 0.097 & 0.267 & 0.538 & 0.195 & 0.000337 & 0.00068 & 0.000246 \\
\hline & PS & 0.212 & 0.168 & 0.091 & 0.123 & 0.32 & 0.557 & 0.000396 & 0.001034 & 0.0018 \\
\hline & WR & 0.212 & 0.052 & 0.073 & 0.11 & 0.346 & 0.544 & $8.85 \mathrm{E}-05$ & 0.000278 & 0.000436 \\
\hline & SP & 0.212 & 0.085 & 0.092 & 0.198 & 0.312 & 0.49 & 0.000329 & 0.000519 & 0.000816 \\
\hline \multirow{4}{*}{$\begin{array}{l}\text { Electrical and } \\
\text { controls }\end{array}$} & ED & 0.059 & 0.453 & 0.277 & 0.531 & 0.322 & 0.147 & 0.003958 & 0.002402 & 0.001093 \\
\hline & HD & 0.059 & 0.261 & 0.159 & 0.312 & 0.49 & 0.198 & 0.000771 & 0.001212 & 0.000488 \\
\hline & $\mathrm{CC}$ & 0.059 & 0.168 & 0.303 & 0.539 & 0.297 & 0.164 & 0.00163 & 0.000899 & 0.000495 \\
\hline & $\mathbf{C H}$ & 0.059 & 0.117 & 0.262 & 0.557 & 0.32 & 0.123 & 0.001017 & 0.000585 & 0.000224 \\
\hline \multirow{3}{*}{ Geometric constraints } & SL & 0.092 & 0.478 & 0.375 & 0.137 & 0.239 & 0.623 & 0.002259 & 0.00394 & 0.010254 \\
\hline & DX & 0.092 & 0.172 & 0.225 & 0.142 & 0.334 & 0.525 & 0.000502 & 0.001184 & 0.001861 \\
\hline & NA & 0.293 & 0.35 & 0.4 & 0.123 & 0.32 & 0.557 & 0.00502 & 0.013112 & 0.022811 \\
\hline \multirow{3}{*}{ Reliability } & $\mathbf{P C}$ & 0.293 & 0.594 & 0.318 & 0.304 & 0.172 & 0.524 & 0.01679 & 0.009499 & 0.028906 \\
\hline & $\mathbf{S M}$ & 0.293 & 0.297 & 0.353 & 0.557 & 0.32 & 0.123 & 0.017069 & 0.009811 & 0.003757 \\
\hline & RB & 0.293 & 0.109 & 0.329 & 0.539 & 0.297 & 0.164 & 0.005677 & 0.003131 & 0.001725 \\
\hline \multirow{4}{*}{ Repeatability } & MF & 0.191 & 0.448 & 0.365 & 0.623 & 0.239 & 0.137 & 0.019505 & 0.007495 & 0.004297 \\
\hline & MR & 0.191 & 0.29 & 0.291 & 0.524 & 0.304 & 0.172 & 0.008481 & 0.004926 & 0.002787 \\
\hline & SQ & 0.191 & 0.162 & 0.195 & 0.137 & 0.239 & 0.623 & 0.00083 & 0.001448 & 0.003767 \\
\hline & SR & 0.191 & 0.099 & 0.149 & 0.539 & 0.297 & 0.164 & 0.001532 & 0.000845 & 0.000466 \\
\hline \multirow{4}{*}{ Economic viability } & CT & 0.152 & 0.312 & 0.18 & 0.137 & 0.239 & 0.623 & 0.001177 & 0.002054 & 0.005345 \\
\hline & WT & 0.152 & 0.234 & 0.25 & 0.137 & 0.239 & 0.623 & 0.001224 & 0.002135 & 0.005555 \\
\hline & SA & 0.152 & 0.12 & 0.328 & 0.133 & 0.211 & 0.655 & 0.000804 & 0.001274 & 0.003948 \\
\hline & NW & 0.152 & 0.333 & 0.242 & 0.133 & 0.211 & 0.655 & 0.001642 & 0.0026 & 0.008057 \\
\hline \multirow{2}{*}{\multicolumn{8}{|c|}{$\begin{array}{r}D_{i(S C)} \\
D_{i(S C) \text { Normailized }}\end{array}$}} & 0.095321 & 0.075974 & 0.110861 \\
\hline & & & & & & & & $\underline{0.33783}$ & 0.269262 & 0.392907 \\
\hline
\end{tabular}

Note that, $P_{j(S C)}$ (column iii) is obtained from Table $3 ; A_{k j(S C)}^{D}$ (column iv) for dimension configuration of robots mapped along with the determinant scope of customization is obtained from Table 4 and can be similarly obtained for other dimensions, namely, electrical and controls, geometric constraints, etc.; $A_{k j(S C)}^{I}$ (column v) is obtained from the Table 8; $S_{i k j(S C)}$ (column vi, vii and viii) is obtained from Table 6 for $i=\mathrm{A}, \mathrm{B}$ and $\mathrm{C}$ respectively. The columns ix, $\mathrm{x}$ and $\mathrm{xi}$ correspondingly give the products $P_{j(S C)} A_{k j(S C)}^{D} A_{k j(S C)}^{I} S_{i k j(S C)}$ for $i=\mathrm{A}, \mathrm{B}$ and C. The individual summations are then taken to determine the desirability indices of determinant Scope of customization for the alternatives A, B and C. Finally the normalized desirability indices are determined which are shown in Table 9.

The values indicate that robot supplier $\mathrm{C}$ with a normalized desirability index of 0.3929 has maximum influence on scope of customization availability to the end-user. Supplier C is followed by robot supplier A which has a normalized index of 0.3378 which is followed by the supplier B having a normalized 
desirability index of 0.2693 and hence has least scope of customization available to the customer.

\subsection{Overall Weighted Index and Selection of Best Alternative}

The physical interpretation of the values and desirability indices obtained in the previous section are that if the availability of the scope of customization is the only criteria for selection of robotic supplier then the decision makers will opt for supplier $\mathrm{C}$ which has higher desirability index corresponding to the scope of customization rather then suppliers A and B which have lesser desirability index.

However, note that, in the model at hand the selection of robot supplier is subject to five different determinant level attributes. Table 7 presents desirability indices matrix for the alternatives with respect to only one determinant, namely, scope of customization. Next step should be the determination of desirability matrices for the alternatives with respect to other determinants, namely, worth, quality, performance and compatibility with existing set-up at customer site. Similar matrices and priority vectors as generated from Table 3 to Table 8 to obtain the respective desirability indices for all the determinants. For brevity, the detailed calculations for the four determinant attributes are not shown here and the normalized desirability indices are directly shown in Table 10 . The desirability indices for all the five determinants along with the priority vectors (weighted values) from the pairwise comparison matrices obtained in Table 2 are then used to determine the overall weighted index (OWI) for the alternatives. The OWI for an alternative is obtained as:

$$
O W I=\sum D_{i a N} C_{a}: i=\mathrm{A}, \mathrm{B} \text { and } \mathrm{C}
$$

where $D_{i a N}$ are the normalized desirability indices calculated for the each determinant and the $C_{a}$ are the relative importance weights of the determinants among each others, obtained using the pairwise comparison matrices. In the calculation of OWI the use of normalized values of Dia ensures that the OWI values of the alternatives do not change with a large range of absolute values of Dia for different determinants.

The OWI, calculated from the $D_{i a N}$ and $C_{a}$, for the three alternative robotic suppliers A, B and C are presented in Table 10. The final selection is now done based upon the OWI obtained in the Table 8 . The results from Table 10 indicate that robotic supplier A has the highest OWI (0.3471) as compared to B and C

Table 8: Overall Weighted Index (OWI) for the Alternatives

\begin{tabular}{|c|c|c|c|c|c|c|}
\hline \multirow{3}{*}{$\begin{array}{c}\text { Robot } \\
\text { Suppliers }\end{array}$} & $\begin{array}{c}\text { Scope of } \\
\text { Customization } \\
\end{array}$ & Worth & Quality & Performance & Compatibility & \multirow{3}{*}{ OWI } \\
\hline & \multicolumn{5}{|c|}{ Priority vector (e-values) } & \\
\hline & 0.1981 & 0.1282 & 0.0846 & 0.2142 & $\underline{0.3749}$ & \\
\hline $\mathbf{A}$ & 0.3378 & 0.2987 & $\overline{0.4424}$ & 0.3952 & 0.3254 & 0.3471 \\
\hline B & 0.2693 & 0.2687 & 0.3235 & 0.3269 & 0.3327 & 0.3085 \\
\hline C & 0.3929 & 0.4326 & 0.2341 & 0.2779 & 0.3419 & 0.3441 \\
\hline
\end{tabular}

who have 0.3085 and 0.3441 respectively. Many managerial implications can also be drawn from the analysis. For instance, robotic supplier A must note that while its OWI is maximum as compared to $\mathrm{B}$ and $\mathrm{C}$, however with respect to the determinants scope of customization, worth and compatibility its score (desirability indices) are lower than that of B and C. hence, A needs to emphasis on these aspects. Significantly, the desirability indices of $\mathrm{C}$ with respect to quality determinant is quite low as compared to its competitors and hence its overall OWI is also affected. The robotic supplier should emphasis on the quality of its robots to improve its product desirability by the customer.

The ANP methodology, thus, provides a scientific tool for decision making in selection of robot supplier in particular and multiple attribute decision environment in general.

\section{Comparisons of Decision Results Using ANP and Other Efficient MADM Tool-AHP}

In this section, the decision results for robot selection using the analytical network process (ANP), as presented in Section 3, are compared with the results obtained using another efficient tool for decision making in multiple attribute environment-analytical hierarchal process (AHP). Indeed AHP is a special case of ANP wherein the intra-attributes influence do not exist. Moreover, the attributes at lower level do not influence the attributes and selection parameters at higher levels. Specifically, the attributes are arranged in hierarchy rather than network. Note that, for the purpose of valid comparisons same selection criteria and influencing attributes are considered as in ANP model.

\subsection{Robot Selection Using the AHP Technique and AHP Model Formulation}

The results of robot selection using AHP are now compared with those obtained using the selection based on ANP as presented in previous section. The robot selection process is broken into a hierarchy of interrelated decision elements that include the criteria, sub-criteria, and robots. Thus determinant level, dimension level and facilitator level attributes are identified and put in the

hierarchy, as shown in Figure 4. Since, unlike ANP, in AHP there is no influence of attributes

on same level of hierarchy among each other, the levels are named as level-1, level-2 and level-3 attributes. s previously mentioned, the attributes influencing the robot selection are taken same as those in ANP model. However, the intra-attributes influences are mapped in hierarchal order only.

\subsubsection{Pair-wise Comparison of Decision Elements at Level-3 and Evaluation of Weighted Importance of Alternatives at Level-3}

The pair wise comparisons of the decision elements at level-3 of the hierarchy are done by each of the decision makers. Thus, the pair wise comparisons of these alternative suppliers (namely, A, B and $\mathrm{C}$ ) were done to map their relative impact on each of the facilitator level (Level-3) attribute. The methodology of collection of numerical ratings is based on the Saaty's table and the procedure as mentioned in Section 2.3. Since, there are thirty attributes at level-3, and pair-wise comparisons of $\mathrm{A}, \mathrm{B}$ and $\mathrm{C}$ is done with respect to each of the level- 3 attributes, there will be thirty matrices in total. These are presented in Appendix A1. Note that, similar pair wise comparison matrices are available from Section 2.7, Table 6. However, the pair wise matrices in AHP (matrices given in Appendix A1) will be different from those in table 6 and Section 2.3 because pair wise comparisons in Section 2.3 are done under the influence of determinants on the controlling criteria. Thus, table 6 gives pair wise comparison matrices for alternatives' impact on enabler Payload capacity (PC) in influencing the Scope of customization determinant, while the matrices in AHP give overall impact of alternatives on controlling criteria of payload capacity. Evidently, ANP tracks more intricate dependencies and hence is more accurate. The priority vectors 
from these matrices are then taken for hierarchal evaluation at next level.

The next step is the evaluation of the combined effect of the relative weights of decision elements at level-3 on alternatives. For this, first the pair wise comparison matrices for the level-3 attributes are generated. Since, there are six clusters of hierarchal chain as shown in figure 2, attributes at level-3 are compared pairwise in six different clusters. Thus, six matrices are formed. Note that, these matrices will be different from those in Section 2.5 as the pair wise comparisons of facilitator level attributes in section 2.5 map the combined effect of configuration of root under scope of customization determinant. In the AHP overall pair wise comparisons of these clustered attributes at level-3 is done, irrespective of the controlling dimensions and the determinants. One such matrix for the cluster belonging to configuration of robot dimension is shown in Table 9.

Table 9: Pairwise comparisons of facilitator level attributes in cluster of configuration of root

\begin{tabular}{|c|c|c|c|c|c|c|c|c|c|c|c|c|c|}
\hline & PC & WS & OC & $\mathrm{AC}$ & RS & $\mathrm{CE}$ & MA & JT & PR & PS & WR & SP & PV \\
\hline PC & 1 & 3 & 2 & 2 & 3 & 5 & $1 / 4$ & $1 / 3$ & 3 & $1 / 4$ & $1 / 2$ & $1 / 2$ & 0.083 \\
\hline WS & $1 / 2$ & 1 & $1 / 3$ & 3 & $1 / 4$ & 2 & $1 / 2$ & $1 / 2$ & 4 & $1 / 4$ & $1 / 4$ & $1 / 3$ & 0.0521 \\
\hline OC & 2 & 2 & 1 & 3 & 3 & 2 & $1 / 2$ & 2 & 5 & $1 / 4$ & 4 & 4 & 0.1198 \\
\hline RS & $1 / 2$ & 2 & $1 / 2$ & $1 / 2$ & 1 & 4 & $1 / 2$ & $1 / 4$ & 4 & $1 / 4$ & 3 & $1 / 4$ & 0.0622 \\
\hline CE & $1 / 4$ & $1 / 3$ & $1 / 4$ & $1 / 4$ & $1 / 4$ & 1 & $1 / 2$ & $1 / 4$ & $1 / 4$ & $1 / 2$ & $1 / 2$ & $1 / 4$ & 0.0274 \\
\hline MA & 3 & 4 & 1 & 4 & 3 & 4 & 1 & 2 & 2 & 2 & 3 & 4 & 0.1563 \\
\hline JT & 3 & 2 & $1 / 3$ & 2 & 3 & 3 & $1 / 2$ & 1 & 2 & $1 / 2$ & $1 / 2$ & 3 & 0.0878 \\
\hline PR & $1 / 2$ & $1 / 3$ & $1 / 4$ & $1 / 2$ & $1 / 2$ & 4 & $1 / 2$ & 1 & 1 & $1 / 2$ & 3 & 4 & 0.0683 \\
\hline PS & 4 & 3 & 2 & 4 & 4 & 4 & $1 / 2$ & 3 & 4 & 1 & 5 & 2 & 0.1614 \\
\hline WR & 2 & 3 & $1 / 3$ & $1 / 3$ & $1 / 2$ & 4 & $1 / 4$ & $1 / 2$ & $1 / 2$ & $1 / 4$ & 1 & $1 / 4$ & 0.0494 \\
\hline Sp & 2 & 4 & $1 / 3$ & 3 & 3 & 4 & $1 / 3$ & $1 / 2$ & $1 / 3$ & $1 / 2$ & 2 & 1 & 0.0814 \\
\hline
\end{tabular}

Table 10: Weighted Importance for the Alternatives at level-3 attributes

\begin{tabular}{|c|c|c|c|c|c|c|c|c|c|c|c|c|c|}
\hline \multirow{3}{*}{$\begin{array}{c}\text { Robot } \\
\text { Suppliers }\end{array}$} & $\mathbf{P C}$ & WS & $\mathbf{O C}$ & $\overline{\mathbf{A C}}$ & $\mathbf{R S}$ & $\mathbf{C E}$ & MA & JT & $\mathbf{P R}$ & PS & WR & $\mathbf{S P}$ & \multirow{3}{*}{ Index 3} \\
\hline & \multicolumn{12}{|c|}{ Priority vector (e-values) from Table 11} & \\
\hline & 0.0832 & 0.0521 & 0.1198 & 0.0509 & 0.0622 & 0.0274 & 0.1563 & 0.0878 & $\mathbf{0 . 0 6 8 3}$ & 0.1614 & 0.0494 & 0.0814 & \\
\hline $\mathbf{A}$ & 0.3803 & 0.5247 & 0.5571 & 0.2431 & 0.1925 & 0.5536 & 0.2114 & 0.2766 & 0.2508 & 0.1185 & 0.0964 & 0.163 & 0.4167 \\
\hline B & 0.5013 & 0.3338 & 0.3202 & 0.6389 & 0.6768 & 03135 & 0.6551 & 0.5949 & 0.59 & 0.5013 & 0.2831 & 0.2969 & 0.2675 \\
\hline $\mathbf{C}$ & 0.1185 & 0.1416 & 0.1226 & 0.1181 & 0.1307 & 0.1329 & 0.1335 & 0.1285 & 0.1591 & 0.3803 & 0.6205 & 0.6401 & 0.3158 \\
\hline
\end{tabular}

The priority vectors from these six clustered pairwise comparison matrices of facilitators at level-3 is now taken for evaluation of overall weighted importance of attributes at level-3. The step for the twelve attributes in cluster configuration of robot is shown here in Table 10. The weighted importance number at the level-3 attributes is obtained as:

$$
\text { Index3 }=\sum E_{i a N} e_{a}: i=\mathrm{A}, \mathrm{B} \text { and } \mathrm{C}
$$

where $E_{i a N}$ are the normalized desirability indices calculated for the each enabler/facilitator level selection criteria/attribute and $e_{a}$ are the relative importance weights of these attributes among each others, obtained using the pairwise comparison matrices (Table 9). Thus a set of six pairwise indices are obtained. These indices will be used for the weightage determination of alternatives at level-2 attributes. This is presented in next sub-section.

\subsubsection{Pair-wise Comparison of Attributes at Level-2 and Evaluation of Its Weighted Importance of Alternatives at Level-2}

Next the weighted importance of various attributes at level-2 are determined. For this, the pairwise comparison matrices for attributes at dimension level (level-2) are required. These are generated by taking ratings from the decision makers using the methodology presented in Section 3.3. Since, there are five level-1 attributes (determinants) and six level-2 attributes (dimensions) there will be five such pairwise comparison matrices. One such matrix giving the pairwise comparison of dimension (level-2) attributes with respect to scope of the customization available for end-user is presented in Table 11. Again, the matrix in Table 11 is different from matrix in Table 3 as the pairwise comparisons shown in cluster level attributes in Table 3 is when determinants are also the controlling parameters.

Table 11: Pairwise comparisons of cluster level attributes (determinant: Scope of customization)

\begin{tabular}{|c|c|c|}
\hline & CR & EC \\
\hline Configuration of robot (CR) & 1 & 2 \\
\hline Electrical and Controls (EC) & $1 / 2$ & 1 \\
\hline Geometric constraints (GC) & $1 / 3$ & 3 \\
\hline Repeatability (RP) & 4 & 3 \\
\hline Reliability (RL) & $1 / 3$ & 4 \\
\hline Economic Viability (EV) & $1 / 2$ & 3 \\
\hline
\end{tabular}

In total five such matrices are formed, one with respect to each determinant level attribute. Next, the combined effect of the relative weights of decision elements at level-2 on alternatives is evaluated. The weighted indices for the alternatives from the previous step are taken and weighted index of impact of level-2 criteria on the alternatives is determined using the equation:

$$
\text { Index } 2=\sum F_{i a N} f_{a}: i=\mathrm{A}, \mathrm{B} \text { and } \mathrm{C}
$$

\begin{tabular}{|c|c|c|c|c|}
\hline GC & RP & RL & EV & Priority vector \\
\hline 3 & $1 / 4$ & 3 & 2 & $\mathbf{0 . 2 0 8 3}$ \\
\hline $1 / 3$ & $1 / 3$ & $1 / 4$ & $1 / 3$ & $\mathbf{0 . 0 5 9 4}$ \\
\hline 4 & $1 / 4$ & $1 / 2$ & $1 / 4$ & $\mathbf{0 . 0 8 3 2}$ \\
\hline 2 & 1 & 2 & 2 & $\mathbf{0 . 3 2 6 5}$ \\
\hline 4 & $1 / 2$ & 1 & 2 & $\mathbf{0 . 1 6 9 5}$ \\
\hline
\end{tabular}

where $F_{i a N}$ are the normalized desirability indices calculated for the each dimension/cluster level attribute and $f_{a}$ are the relative importance weights of these dimensions among each others, obtained using the pairwise comparison matrices (Table 11).

The Table 12 shows the determination of weighted impact of the level-2 attributes on the alternatives, A, B and C for the scope of customization controlling criteria.

Table 12: Weighted Importance of alternatives at level-2 attributes for scope of customization

\begin{tabular}{|c|c|c|c|c|c|c|c|}
\hline $\begin{array}{c}\text { Robot } \\
\text { Suppliers }\end{array}$ & $\begin{array}{c}\text { Configuration of } \\
\text { robot }\end{array}$ & $\begin{array}{c}\text { Electrical and } \\
\text { controls }\end{array}$ & $\begin{array}{c}\text { Geometric } \\
\text { constraints }\end{array}$ & Repeatability & Reliability & $\begin{array}{c}\text { Economic } \\
\text { viability }\end{array}$ & Index2 \\
\hline & \multicolumn{7}{|c|}{ Priority vector (e-values) from Table 13 } \\
\hline
\end{tabular}




\begin{tabular}{|l|l|l|l|l|l|l|l|}
\hline & $\mathbf{0 . 2 0 8 3}$ & $\mathbf{0 . 0 5 9 4}$ & $\mathbf{0 . 0 8 3 1}$ & $\mathbf{0 . 3 2 6 4}$ & $\mathbf{0 . 1 6 9 5}$ & $\mathbf{0 . 1 5 3 0}$ & \\
\hline A & 0.4167 & 0.3325 & 0.2423 & 0.3937 & 0.2751 & 0.4221 & $\mathbf{0 . 3 6 6 4}$ \\
\hline B & 0.2675 & 0.2514 & 0.3105 & 0.2041 & 0.2599 & 0.2261 & $\mathbf{0 . 2 4 1 7}$ \\
\hline C & 0.3158 & 0.4161 & 0.4472 & 0.4022 & 0.465 & 0.3518 & $\mathbf{0 . 3 9 1 6}$ \\
\hline
\end{tabular}

The physical interpretation of the weighted importance of alternatives at level-2 is that if the scope of customization available to the end user is the only criteria of selection at the determinant level, then the robot supplier $\mathrm{C}$, having the highest weighted score of 0.3916 is the best choice. Supplier C is followed by robot suppliers A and B which have weighted scores of 0.3664 and 0.2417 respectively. If these results are compared with those from the ANP analysis, after Table 9 which gives alternatives desirability indices for scope of customization, it is observed that the trends of results are similar. Thus, both the techniques lead to the conclusion that with respect to customization scope the robot supplier $\mathrm{C}$ has highest ratings followed by $\mathrm{A}$ and $\mathrm{B}$. However, with AHP analysis the demarcation between the alternatives ratings is more prominent, while with ANP the results are distributed more uniformly. The ANP analysis suggests that the differences in the OWI may not be as significant as indicated by the AHP analysis if the interdependencies among the selection criteria across the network are also taken into consideration appropriately. The impacts of the analysis of the inter attribute dependencies lead to more cohesive and mature decision making. Thus ANP is a more effective tool in robot selection as compared to AHP. However, note that, AHP is computationally more fast than ANP.

\subsubsection{Pair-wise Comparison of Attributes at Level-1 and Evaluation of Overall Weighted Indices for the Alternatives}

Finally, the influence of the alternatives on various determinants is evaluated to get the overall weighted indices for the alternatives.
Note that, the pair-wise comparison matrix for the various determinants to map their influences on the OWI of alternatives is already available from the Table 2 of ANP analysis. Table 10 presents desirability indices (index-2) for the alternatives with respect to determinant scope of customization. Next step should be the determination of these indices for the other determinants, namely, worth, quality, performance and compatibility with existing set-up at customer site. For brevity, the detailed calculations for the four determinant attributes are not shown here and the normalized weighted indices for it are directly shown in Table 15. The weighted indices for all the five determinants along with the priority vectors (weighted values) from the pairwise comparison matrices obtained in Table 2 are then used to determine the overall weighted index (OWI) for the alternatives. The OWI for an alternative is obtained as:

$$
O W I=\sum H_{i a N} h_{a}: i=\mathrm{A}, \mathrm{B} \text { and } \mathrm{C}
$$

where $H_{i a N}$ are the normalized weighted indices calculated for the each determinant and the $h_{a}$ are the relative importance weights of the determinants among each others, obtained using the pairwise comparison matrices (Table 2). The OWI, calculated from the $H_{i a N}$ and $h_{a}$, for the three alternative robotic suppliers A, B and C are presented in Table 13.

Table 13: Overall Weighted Index (OWI) for the Alternatives from AHP analysis

\begin{tabular}{|c|c|c|c|c|c|c|}
\hline \multirow{3}{*}{$\begin{array}{c}\text { Robot } \\
\text { Suppliers }\end{array}$} & $\begin{array}{c}\text { Scope of } \\
\text { Customization }\end{array}$ & Worth & Quality & Performance & Compatibility & \multirow{3}{*}{ OWI } \\
\hline & \multicolumn{5}{|c|}{ Priority vector (e-values) } & \\
\hline & 0.1946 & 0.1541 & 0.0761 & 0.2099 & 0.3651 & \\
\hline $\mathbf{B}$ & 0.2417 & 0.2826 & 0.3278 & 0.354 & 0.3312 & $\mathbf{0 . 3 1 0 7 5 5}$ \\
\hline $\mathbf{C}$ & 0.3919 & 0.3957 & 0.2711 & 0.2335 & 0.3638 & $\overline{0.339707}$ \\
\hline
\end{tabular}

\subsection{Comparisons of Results on Robot Alternative Selections from ANP and AHP Techniques}

It is seen that while the trends from the ANP and AHP techniques are similar, there is a higher reliability in the results from ANP

analysis, as it maps the interdependencies among the various criteria more appropriately than in AHP. This mapping is reflected in convergence of the overall weightage indices for the three alternatives, $\mathrm{A}, \mathrm{B}$ and $\mathrm{C}$ as obtained from ANP. It is noted that the differences among OWI of $\mathrm{A}, \mathrm{B}$ and $\mathrm{C}$ from the AHP are more pronounced (Table 14) as compared to the OWIs obtained from ANP analysis (Table 10). While both the techniques provide the results that the robotic selection alternative $\mathrm{A}$ has highest overall rating than the other two alternatives, B and C; the ANP suggests that the differences in the OWI may not be as significant as indicated by the AHP analysis if the interdependencies among the selection criteria across the network are also taken into consideration appropriately. The Overall weighted indices OWI of C from AHP is 0.3397 as compared to OWI of 0.3493 of A (difference $=0.0096$ ). . Considering the impact of interdependencies of attributes and criteria in ANP the difference in OWIs of $\mathrm{C}$ and $\mathrm{A}$ is reduced to 0.0030 .Consequently, selection of supplier $\mathrm{C}$ will not be as bad a preposition as told by AHP analysis Using the ANP analysis there is a $69 \%$ increase in the confidence in the selection of robot C. Thus, ANP provides for higher and better managerial implications than AHP.

Thus, it is clear that ANP gives more refined results for better decision making than the AHP. This is because in ANP the final OWI are determined from the analysis based upon super matrix and limit super matrix. Moreover, the synthesis of inter cluster dependencies among the attributes leads better mapping of the relative importance of the various clusters and attributes.

It must be noted that ANP is computationally slower than the AHP technique because computationally intensive steps like determination of super matrix and limit super matrix are avoided in AHP. However, AHP can not be relied upon in the MADM environments where there exist very strong intra-attributes dependencies. In such cases ANP can give the desirable results. ANP is particularly useful when there exist influences within the clusters and between the alternatives. Indeed, AHP can be shown as a special case of ANP and hence all the advantages associated with AHP are automatically associated with ANP.

\section{Sensitivity Analysis of the Robot Selection Results from ANP and AHP Techniques}

The purpose of the sensitivity analysis is to analyze the variations in the results (OWI) for the alternatives with respect to the changes in the ratings or importance assigned to the attributes at 
hand. It is important for the decision makers to track the changes in the final weight age of the alternatives with a subjective variation in ratings of the attributes. Moreover, such an analysis will also help in estimation of importance/efforts required to be attached with a particular attribute to increase the final rating of robot vis-à-vis competitors' robot. Similarly, it is beneficial to know weather or not the strategy adopted for a particular attribute is over emphasized or biased in a particular direction. This will help in identification of the buffer or scope available for reduction in emphasis attached to an attribute at hand while still maintaining a higher OWI as compared to the competitors. Sensitivity analysis also helps in increasing the confidence level of decision makers on the overall ratings given by them and results from a particular tool at hand. In this section, Sensitivity analysis of the results, i.e. OWI of the alternatives, obtained using ANP and AHP is carried out. The comparisons of sensitivity analysis of results from ANP and AHP also further highlight the advantages of higher resolution of results obtained from decision making using ANP technique. The analysis is done with respect to change in the ratings and importance attached to the determinant level attributes only, although the similar exercise can be easily extended to map the influence on the OWI due to change in ratings of any attribute, at any level.

\subsection{Variation in the OWI with Change in Weightage of Cost v/s Quality}

It was identified in Section 3 from the ANP results analysis that robotic supplier $\mathrm{C}$ has very low desirability indices with respect to quality attributes. Similarly, with respect to cost attributes the desirability indices of robotics supplier A is quite low. Thus, both these suppliers must be interested in knowing the changes in the OWI with variation in the weightage of cost verses quality. The question raised is, "What will the OWI be if quality is given $x$ rating with respect to cost considerations, and $\mathrm{x}$ varies from 1 to 10 ?" The sensitivity results are shown in Figure 5.

Fig. 5: Variation in priority of robots and robot suppliers with changes in weights assigned to cost with respect to quality

It is clear that the OWI of robot supplier $\mathrm{C}$ will become more than that of $\mathrm{A}$ if a weightage of more than 6 is assigned to cost with respect to the quality. The interpretation for $\mathrm{A}$ can be that it has only a marginal advantage over robot $\mathrm{C}$ with respect to quality and if a higher weightage is assigned to the cost than quality A will loose its OWI upper-hand over C. Similar analysis can be extended to robot (and its supplier) B.

It must be noted that the trends in sensitivity analysis from AHP do not present this picture. Instead, as per the AHP based sensitivity analysis the OWI of A will remain higher than that of B and $\mathrm{C}$ even if highest weightage is assigned to cost than the quality. Indeed, the ANP based results lead to better managerial implications and advantages of adopting ANP technique over AHP for the MADM environment of robot selection is once again highlighted.

\subsection{Other Sensitivity Analysis Results}

Figure 6(a-c) presents the sensitivity analysis for the OWI with the variation in weightage to the quality and performance, cost with respect to performance, and cost with respect to scope of customization respectively. Similar analysis can be extended to any combination of the parameters and the affect for variation in the assignment of weightage to one selection criteria with respect to the other criteria can be studied.

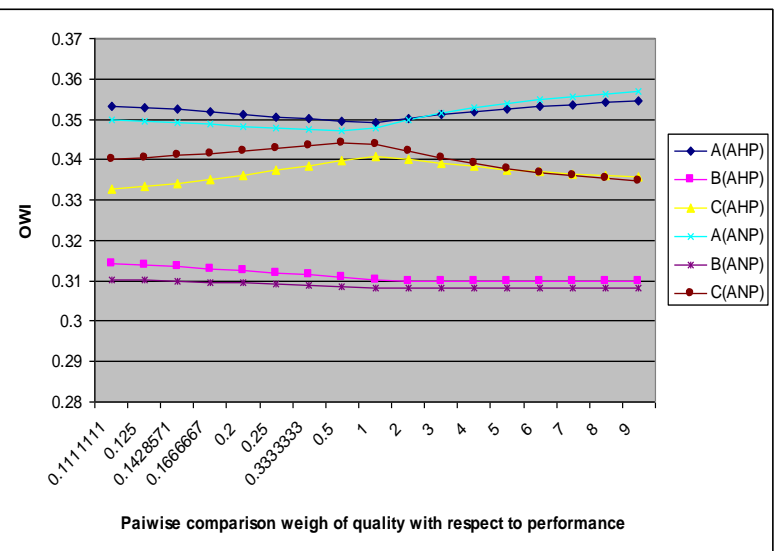

(a) Quality with respect to performance
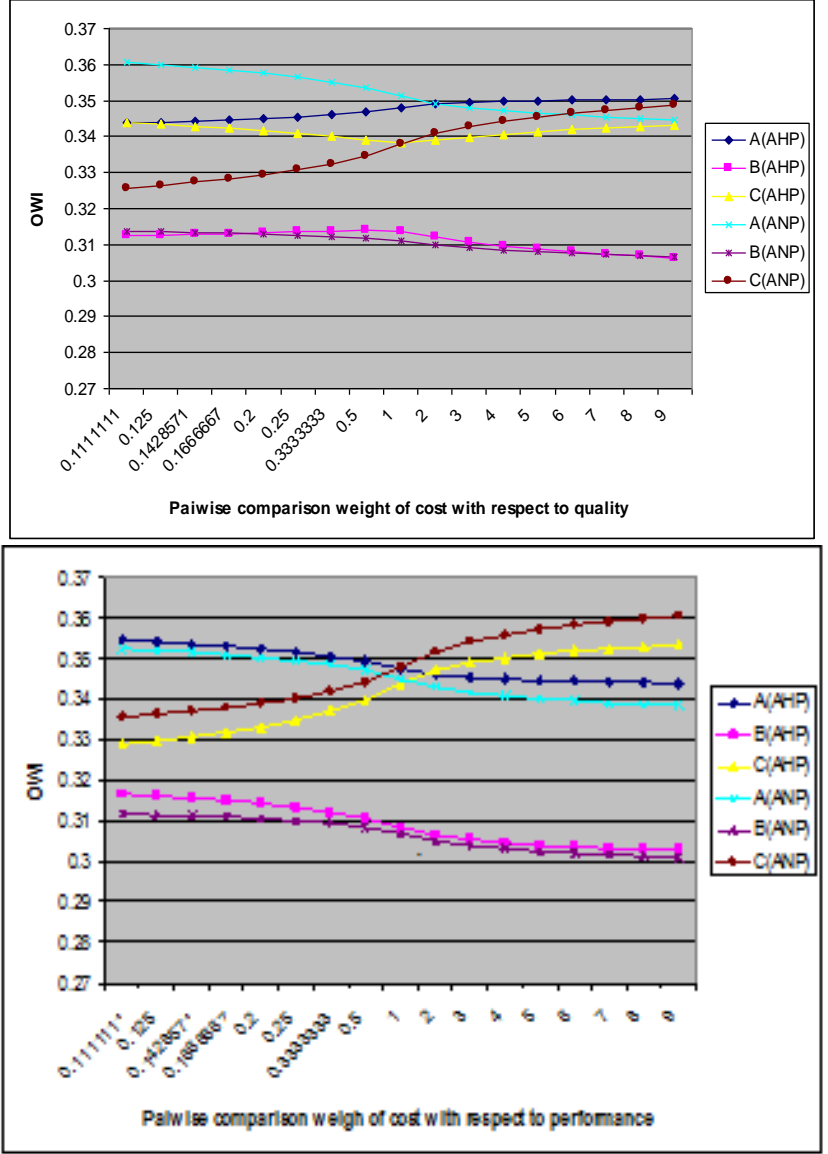

(b) Cost with respect to performance

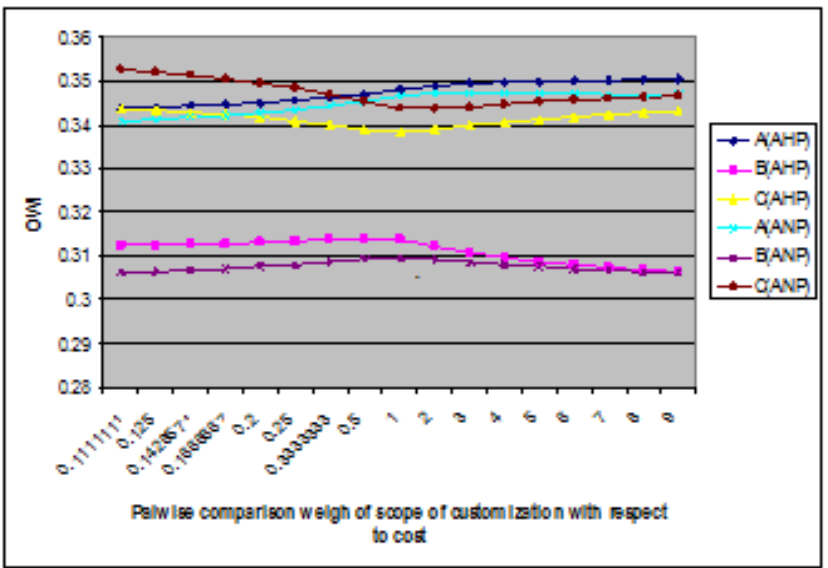

(c) Scope of customization with respect to cost

Fig. 6: Variation in priority of robots and robot suppliers with changes in pair wise weightage assignment to various selection criteria 


\section{Conclusion}

At a time when robotics based automation is changing the manufacturing practices in most of the industries, this paper provides an insight of the various aspects of robot selection decision making. Various attributes influencing the robot section are identified. The attributes are then grouped in clustered and networking is done. Mapping of the clusters of the attributes is done to trace the inter-cluster influences. Next, the sub-elements and attributes are mapped for their intra-dependencies. The ANP is applied to the robot selection process and finally a sequence of priority of the attributes is obtained. Payload and workspace considerations are identified as the most important selection attributes. However, it is also pointed out that ANP, like AHP, is a quantitative assessment tool and hence the decision making procedure is also subjective. The sensitivity analysis on the overall weighted indices to study the affects of assignment of different weightage to various selection criteria in pairwise comparisons on OWI is also carried out. The advantages of ANP over AHP as decision making technique for robot selection in MADM environment are highlighted. A brief survey of various trends in the decision making techniques in production management is also presented and it is shown that researchers are striving to achieve highest computational efficiency and versatility in the decision making softwares.

\section{References}

[1] R. Hinson, "Robotics-environment a major factor in robot selection," Industrial Engineering, vol. 15 (10), 1983, pp. 32-30.

[2] R. Hinson, "Knowing work envelops helps in evaluating robots," Industrial Engineering, vol. 15 (7), 1983, pp. 22-27.

[3] R. C. Dorf, and S. Y. Nof, Int. Encyclopedia of Robotics Applications and Automation, John Wiley \& Sons, Inc., New York, 1988.

[4] E. I. Rivin, Mechanical Design of Robots, McGraw Hill, NY, 1988.

[5] T. Yoshikawa, Foundations of Robotics Analysis and Control, Presntice-Hall of India Pvt. Ltd., New Delhi, 1998.

[6] K. Knott, and R. D. Getto, "A model for evaluating alternative robot systems under uncertainty," Int J Production Research 1982;20:155-65.

[7] DR Towill, "A Production Engineering Approach to Robot Selection,” Omega Int. J. of Mgmt Sci., Vol. 12, No. 3, pp. 261 272,1984

[8] Bartholomew O. Nnaji and M. Yannacopoulou, "A utility theory based robot selection and evaluation for electronics assembly," Computers ind. Engng Vol. 14, No. 4, pp. 477-493, 1988

[9] M. M. Imang and R. J. Schlesinger, "Decision models for robo selection: a comparison of ordinary least squares and linear goal programming method", Decision Science, 20, pp. 40-53, 1989.

[10] N. Boubekri, M. Sahoui and C. Lakrib, "Development of an expert system for industrial robot selection," Computational Industrial Engineering 1991;20:119-27.

[11] Chao-Yen Wu, "Robot selection decision support system: a fuzzy set approach," Math/ Comput. Mafelling, Vol. 14, pp. 440-443. 1990 ,

[12] M. J. J. Wang," "A decision support system for robot selection," Decision Support Systems 7 (1991) 273-283

[13] V. P. Agrawal, V. Kohli, and S. Gupta, "Computer aided robot selection: the 'multiple attribute decision making' approach," Int $\mathrm{J}$ Production research 1991; 29:1629-44.

[14] R. Venkata Rao and K.K. Padmanabhan, "Selection, identification and comparison of industrial robots using digraph and matrix methods," Robotics and Computer-Integrated Manufacturing 22 (2006) 373-383. 